\title{
Using Registration Uncertainty Visualization in a User Study of a Simple Surgical Task
}

\author{
Amber L. Simpson ${ }^{1}$, Burton Ma ${ }^{1,2}$, Elvis C.S. Chen ${ }^{1}$, Randy E. Ellis ${ }^{1,2,3}$, \\ and A. James Stewart ${ }^{1,2}$ \\ ${ }^{1}$ School of Computing, Queen's University, Kingston, Canada \\ ${ }^{2}$ Human Mobility Research Centre, Kingston General Hospital, Kingston, Canada \\ ${ }^{3}$ Surgical Planning Laboratory, Brigham and Women's Hospital, Boston, USA \\ \{simpson, jstewart\}@es.queensu.ca
}

\begin{abstract}
We present a novel method to visualize registration uncertainty and a simple study to motivate the use of uncertainty visualization in computer-assisted surgery. Our visualization method resulted in a statistically significant reduction in the number of attempts required to localize a target, and a statistically significant reduction in the number of targets that our subjects failed to localize. Most notably, our work addresses the existence of uncertainty in guidance and offers a first step towards helping surgeons make informed decisions in the presence of imperfect data.
\end{abstract}

\section{Introduction}

Computer-assisted surgery all but ignores the visualization of uncertainty in data. Uncertainty might be ignored because of the inherent difficulty in expressing and computing uncertainty during surgery. A second reason could be the lack of meaningful methods of visualizing uncertainty and data. Errors and uncertainty are introduced when data is acquired (through an imaging modality or tracking system), transformed (by registration or segmentation), and rendered (see Figure 1). We contribute a method for visualizing registration uncertainty and a user study that evaluates the visualization method for osteoid osteoma excision.

\subsection{Uncertainty Visualization}

Only a few techniques have been proposed to visualize uncertainty and attempts to quantify the effectiveness of these techniques are virtually nonexistent (see [1] and the references therein). Perhaps this is because it is difficult to define a task that is representative of a typical use that can be statistically analyzed. Methods of visualizing uncertainty in medical applications include brain tumor typing [2], analyzing EEG data of neonatal seizures [3], determining brain fiber orientation in MRI [4], and determining radiation therapy dosing [5]. However, to our best knowledge, no research has investigated the use of uncertainty visualization in computer-assisted surgery.

\subsection{Osteoid Osteoma Excision}

Our study of uncertainty visualization is based on osteoid osteoma excision. An osteoid osteoma is a small, painful, benign bone lesion. In the U.S., osteoid osteomas account 


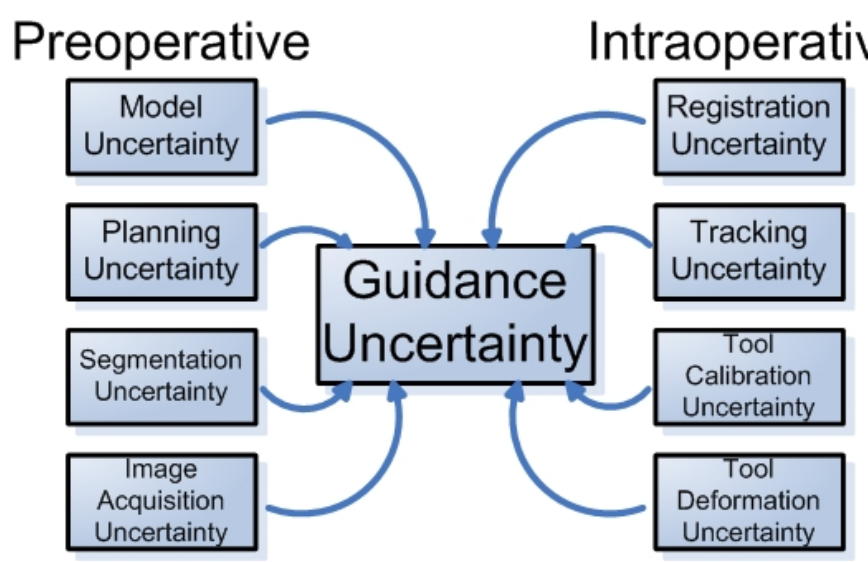

Fig. 1. Illustration of sources of uncertainty in computer-assisted surgery systems. Errors are introduced preoperatively in image acquisition, segmentation, 3D model generation and planning. Intraoperatively, errors occur in registration, tool calibration, tool deformation (deflection) and tracking. Uncertainty from all sources propagates through the entire surgical procedure.

for $12.1 \%$ of benign tumors and $2.9 \%$ of all tumors [6]. In some cases, it may be necessary to remove the tumor. Successful treatment requires complete removal of the nidus surgically. Our affiliated hospital has had success excising these tumors [7] by registering the patient to a pre-operative CT scan and using a tracked drill to expose the nidus.

\section{Methodology}

This section describes how we compute the effect of registration uncertainty on a linear path, visualize the resulting uncertainty distribution, and utilize the visualization in a user study that mimics tumor excision.

\subsection{Registration Uncertainty}

Previous work in registration uncertainty [8] has suggested that the distribution of the registration parameters is approximately symmetric, anisotropic, and leptokurtic (strongly peaked) with heavy tails. In this study, we investigated the distributions of the registration parameters and the resulting effects on a planned linear path. We built an isosurface model of the proximal end of a cadaver femur, and identified a linear path between the lateral cortex of the femur and the medial inferior cortex of the femoral neck such as might be followed in an osteoid osteoma excision (Figure 2). A rapid prototyping machine was used to produce a plastic model of the isosurface model. A calibrated stylus and a Polaris tracking system (Northern Digital Inc., Waterloo, Canada) was used to digitize five distinct landmarks and an additional 50 points from the entire surface of the plastic model; these 55 points were used to compute a good estimate of the ground truth registration transformation $\mathbf{T}_{0}$. To generate registration point sets for learning the 

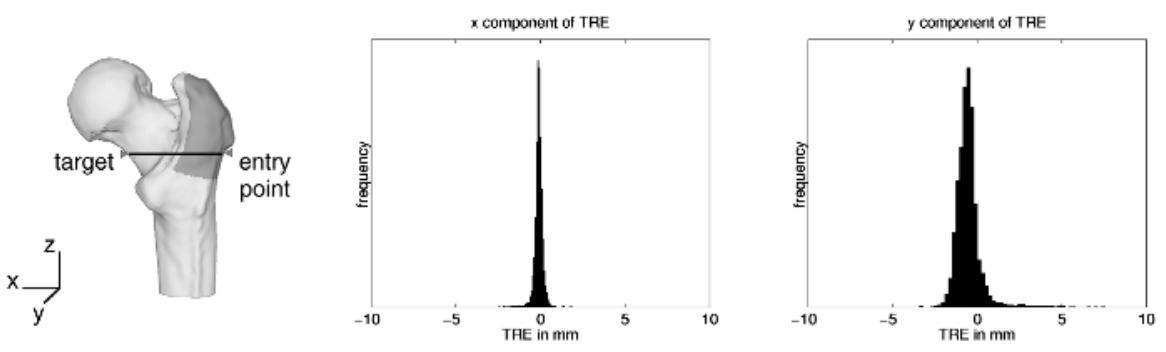

Fig. 2. (Left) Model of the proximal femur showing the registration region in gray and the planned path between the entry point on the lateral cortex and the target point on the medial inferior neck. (Middle and right) The TRE error distribution of the target point under the 10,000 training registrations; the $z$ component of TRE was similar to the $x$ component.

registration parameter distributions, we collected 10 registration points from each of 16 separate regions near the lateral entry point. We generated sets of 16 points each by randomly drawing one point from each of the 16 groups of points; 10,000 sets were generated and registered to the surface model using ICP [9] to produce a set of registration transformations $\left\{\mathbf{T}_{i} \mid i=1 \ldots 10,000\right\}$. A computation of the 10,000 target registration errors (TREs) using the virtual tumor location as the target produced a similar distribution to that observed by Ma and Ellis [8] (Figure2).

\subsection{Visualization Method}

We applied each of the difference transformations $\boldsymbol{\Delta}_{i}=\mathbf{T}_{i} \mathbf{T}_{0}^{-1}$ to the planned path to produce the empirical distribution of paths under registration uncertainty. The simplest visualization technique is to render the paths as individual lines. However, this does not produce an adequate representation of the spatial distribution of the paths.

To convey information about the spatial distribution of the paths, we chose to use volume rendering. With volume rendering, we can view the path distribution as a $3 \mathrm{D}$ volume or as $2 \mathrm{D}$ cross sections, and we can choose what features to accentuate or attenuate by modifying the color and opacity transfer functions.

Our path distribution volume had its long axis aligned with the mean path direction. We computed each slice of the volume at $1 \mathrm{~mm}$ intervals (with slices oriented perpendicular to the long axis) by computing the intersection of every path with the slice. Given that the TRE distribution was approximately symmetric with a significant central spike and broad tails, we fitted a two component mixture of Gaussians to the intersection points on each slice; one Gaussian distribution represented the central spike and the other represented the tails. We used a greedy EM (Expectation-Maximization) algorithm to fit the mixture of Gaussians [10]. Figure 3 illustrates our volume construction method. All of our volume visualizations were performed with the Visualization Toolkit (Kitware Inc., New York, USA) using texture-based volume rendering [11]. Figure 4 shows the volume rendered path distribution from two different views. Figure 5 shows an apparent twisting of the path distribution made apparent by tuning the opacity transfer function. Figure 6 shows the volume and its relationship to the femoral model. 

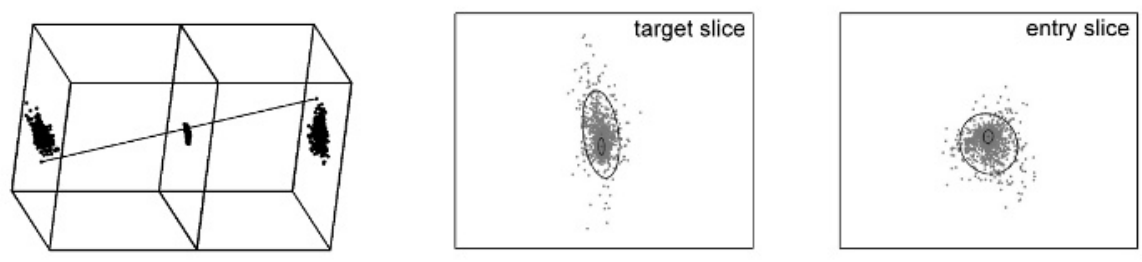

Fig. 3. Illustration of the path distribution volume. (Left) The planned path under each difference transformation $\boldsymbol{\Delta}_{i}$ is intersected with each slice of the volume, and a 2-component mixture of the Gaussians is fit to the intersection points. (Middle and right) Path intersection locations at the target slice (middle) and entry slice (right). The ellipses are centered at the means of the Gaussians; their size and orientation reflect the structure of the covariance matrices. Note the anisotropic distribution at the target location.

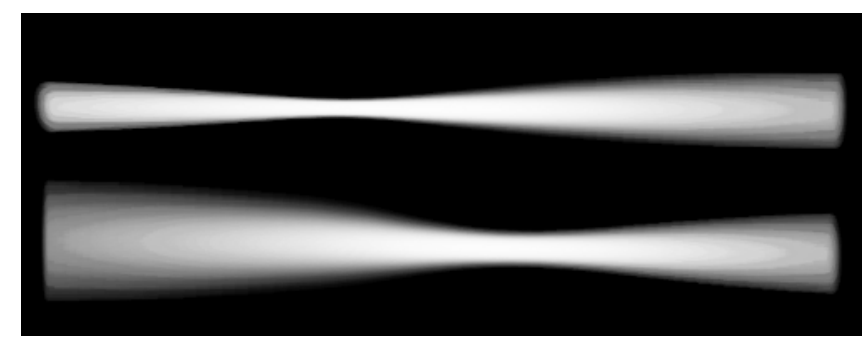

Fig. 4. The volume rendered path distribution from (top) the frontal view and (bottom) the topdown view

\subsection{User Study}

Our study mimics the excision of a deep bone tumor. In this task, the surgeon must expose a blind target (tumor) by drilling through the bone and is immediately aware of success as the tumor is readily visible through the drilled hole. We quantify the success of this task by measuring the number of attempts required to hit the target (tumor).

Hypothesis. Success of instrument placement is better if uncertainty information is visualized and there is sufficient registration error that can cause the subject to miss the target.

Subjects. Fifteen non-expert subjects performed the experiment. The experiment took approximately one hour to complete for each subject. The task was explained to each subject, who was trained using a preliminary trial that consisted of five attempts at each of the two tasks.

Apparatus. Our apparatus consisted of a Polaris tracking system, a calibrated digitizing stylus, a passive dynamic reference body, and an irregular array of 10 metal targets mounted on an optical bench (Figure 7). The flat circular targets were $5 \mathrm{~mm}$ in diameter 

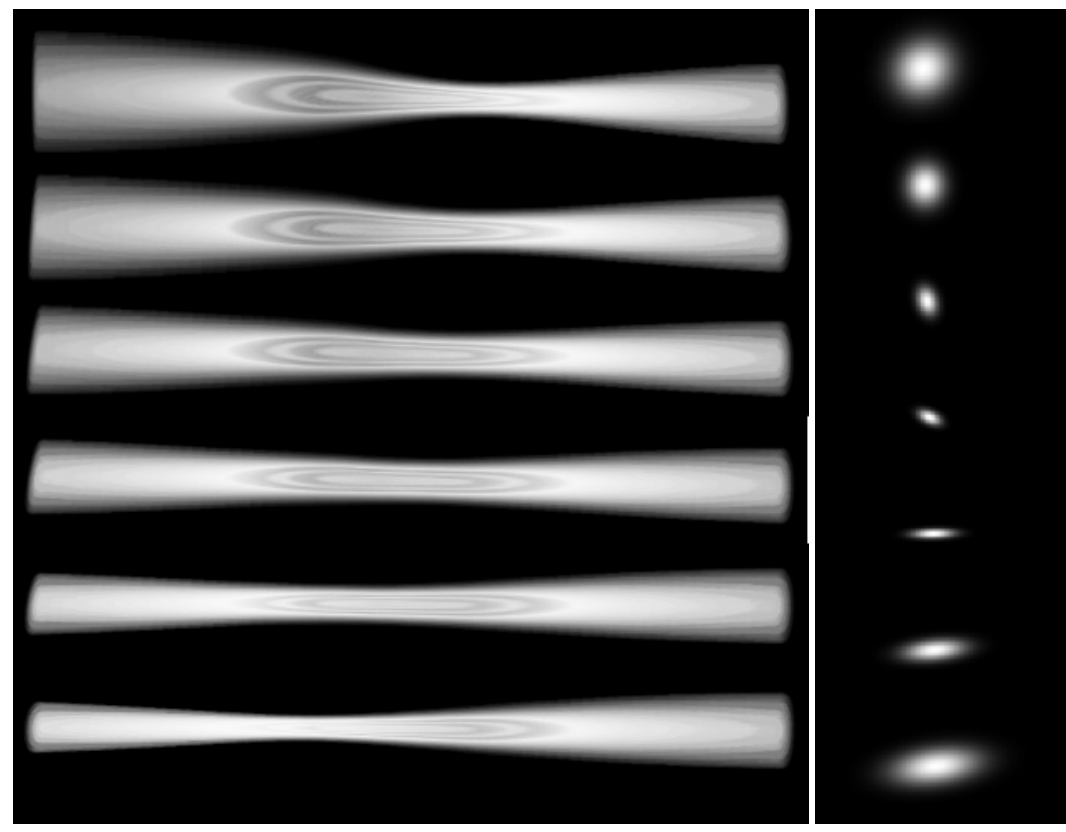

Fig. 5. (Left) Rendering of the path distribution volume from different viewpoints rotating about the long axis of the volume. (Right) Transverse slices through the volume starting at the entry point of the path (top) taken at 10mm intervals along the path until the target (bottom) is reached. Note the twist in the path distribution that would not be evident without visualization of the volume.

and wired to a buzzer that sounded when the stylus touched the target. We placed $2.5 \mathrm{~cm}$ thick modeling clay in front of the target array to hide the targets and to provide support for the stylus as the subjects tried to locate the targets. A computer monitor located in the line of sight of the subject was used to display information to the subject during the experiment.

Stimuli. We performed another 1,000 registrations in a similar way as described in Section 2.1 using a different set of candidate registration points. We randomly selected 10 registrations that produced a TRE of greater than $4 \mathrm{~mm}$ at the target location; these registrations were guaranteed to cause the subject to miss a target if the plan was followed accurately. We emphasize that these registrations were not drawn from the learning distribution used in Section 2.1, nor were they chosen to ensure that they were faithful to the learning distribution; doing either would unfairly bias the results in favor of our hypothesis. These registration transformations were transferred to the array of targets by aligning the planned path on the femoral model to each target.

Subjects were presented with a 3D visualization of a trajectory and point representing the path of the stylus and the location of the target, respectively. Their task was to use the visualization to guide the stylus through the modeling clay to touch the target. The subject repeatedly inserted the instrument into the clay until the target was touched. 


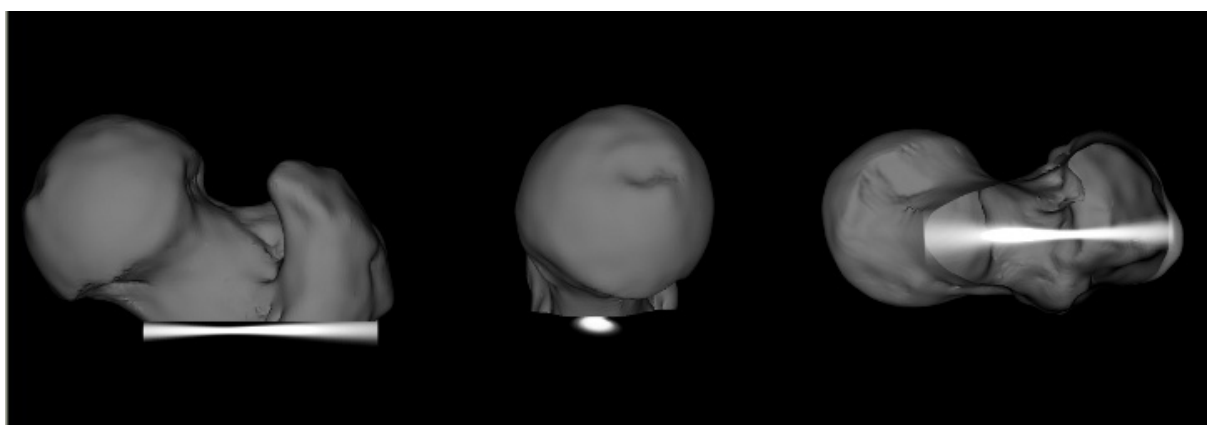

Fig. 6. Model of the proximal femur showing the uncertainty visualization of the planned path between the lateral cortex and the target point (location of tumor) on the medial inferior neck
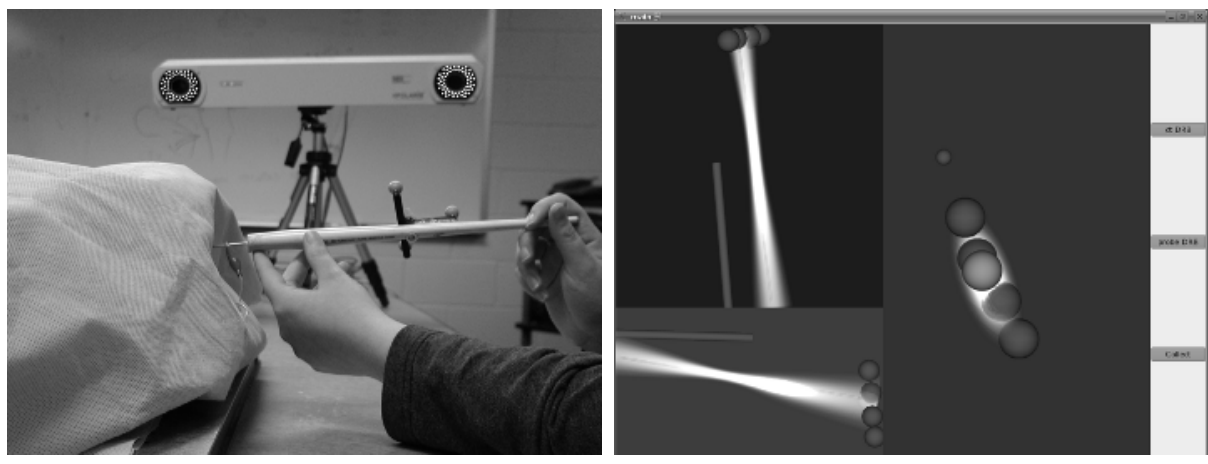

Fig. 7. Illustration of the apparatus. (Left) Subject guiding instrument to target. (Right) Screen capture of stimulus shown to subject. The top-left of the screen is the view along the x-axis (leftright movement of instrument), the bottom-left is the view in the y direction (up-down movement of instrument) and the right of the screen is the view along the $\mathrm{z}$ axis (position of the tip of the instrument).

If the subject failed to localize the target, the instrument was completely withdrawn. Side-to-side movement of the probe was forbidden. The subject was supervised in all tasks. When the subject touched the target successfully, the buzzer sounded. We recorded the number of times it took the subject to touch the target and the position of the tip of the stylus for each missed attempt. The subject was given 10 attempts to locate the target.

Experiment. Each subject was trained using five preliminary trials in order to reduce the effects of learning. Each of the fifteen subjects was presented with 10 tasks without visualizations of uncertainty information and the same 10 tasks with uncertainty information visualized. Within each group of 10, the order in which tasks were presented to subjects was randomized. 


\section{Results}

We measured the number of attempts it took for the subject to touch the target successfully, then analyzed the data using the Wilcoxon signed-ranks test, which is a paired non-parametric test that statistically measures within-subject variability [12]. The Wilcoxon test showed that the number of attempts before success was significantly lower when uncertainty information is visualized $(p=0.0065)$.

We also investigated whether uncertainty information enabled subjects to locate targets that could not be found using ten or fewer attempts. We analyzed the number of incomplete tasks with and without uncertainty information with the Mann-Whitney nonparametric $U$ test, which compares two independent samples [12]. The Mann-Whitney test showed that the visualization of uncertainty significantly reduced the number of incomplete tasks by 1.91 attempts per task $(p=0.0281)$. The total number of incomplete tasks was 43 without uncertainty and 26 with uncertainty.

\section{Discussion}

We demonstrated a simple method to determine the variation caused by registration uncertainty in a planned linear path. We visualized the uncertainty with a path uncertainty volume. Our visualization method resulted in a statistically significant reduction in the number of attempts required to localize a target, and a statistically significant reduction in the number of targets that the pool of subjects failed to localize.

We believe the major source of error in the study is that all subjects were shown the two tasks in the same order, which may have favored the second task. One way to rectify this would be to administer the test to 15 new subjects with the uncertainty visualized first. Note that we elected to limit the number of attempts, which may have underestimated the statistical significance of our results since it is likely that subjects would require more attempts to successfully locate the target. We are currently examining how subjects interpreted our visualizations using the recorded position of the tip of the stylus for each missed attempt.

Visualization of registration uncertainty lets us see unusual and perhaps unexpected features in the distribution of the paths. For example, Figure 4 shows that the distribution has an hourglass shape and that the distribution elongates near the target (see also Figure 3). As another example, Figure 5]demonstrates a twist in the path distribution.

There are many interacting sources of uncertainty in a computer-assisted surgical system and we have only considered registration uncertainty in this article. There are many opportunities for investigating how the various sources of uncertainty affect navigational guidance.

The task defined in our user study may not be representative of how uncertainty would be used in the operating room. Indeed, when we asked a surgical colleague what he would do if he missed an osteoid osteoma in a computer-assisted procedure, his reply was that he would use a bigger drill bit. In practice, we believe that a surgeon would be given a visualization of the uncertainty in the procedure (including all other sources of uncertainty) and decide whether or not to proceed with the procedure as is.

We believe strongly that uncertainty visualization in computer-assisted surgery will help surgeons make more informed decisions in the presence of imperfect data. 
Acknowledgments. This research was supported in part by the Canadian Institutes of Health Research and the Natural Sciences and Engineering Research Council of Canada.

\section{References}

1. David H. Laidlaw et al.: Comparing 2D vector field visualization methods: A user study. IEEE Transactions on Visualization and Computer Graphics 11 (2005) 59-70

2. Vellido, A., Lisboa, P.J.: Handling outliers in brain tumor MRS data analysis through robust topographic mapping. Computers in Biology and Medicine To Appear (2006)

3. N. B. Karayiannia et al.: Quantifying and visualizing uncertainty in EEG data of neonatal seizures. In: Proc of the 26th Annual International Conference of the IEEE EMBS. (2004)

4. Jones, D.K.: Determining and visualizing uncertainty in estimates of fiber orientation from diffusion tensor MRI. Magnetic Resonance in Medicine 49 (2003) 7-12

5. T. McCormick et al.: Target volume uncertainty and a method to visualize its effect on the target dose prescription. International Journal of Radiation Oncology Biology Physics 60 (2004) 1580-1588

6. Dahlin, D.C., Unni, K.: Bone Tumors: General Aspects and Data on 8,542 Cases. 4 edn. Thomas, Springfield, Ill. (1986)

7. Ellis, R.E., Kerr, D., Rudan, J.F., Davidson, L.: Minimally invasive excision of deep bone tumors. In Niessen, W.J., Viergever, M.A., eds.: MICCAI, LNCS \#2208, Springer (2001)

8. Ma, B., Ellis, R.E.: Surface-based registration with a particle filter. In Barillot, C., Haynor, D., Hellier, P., eds.: MICCAI, LNCS \#3216, Springer (2004) 566-573

9. Besl, P., McKay, N.: A method for registration of 3-D shapes. IEEE Transactions on Pattern Analysis and Machine Intelligence 14 (1992) 239-256

10. Vlassis, N., Likas, A.: A greedy EM algorithm for Gaussian mixture learning. Neural Processing Letters 15 (2002) 77-87

11. Fernando, R., ed.: Volume Rendering Techniques. In: GPU Gems: Programming Techniques, Tips, and Tricks for Real-Time Graphics. Addison-Wesley, New York (2004)

12. Sheskin, D.J.: Handbook of Parametric and Nonparametric Tests. 3 edn. Chapman \& Hall, Boca Raton (2004) 